\title{
Tesis doctorales sobre hormigón en universidades españolas: un análisis bibliométrico, 1977-2019
}

\section{Doctoral theses on concrete in Spanish universities: a bibliometric analysis, 1977- 2019}

\author{
Gonzalo Mochón-Bezares $^{(*)}$, Ángela Sorli-Rojo ${ }^{(* *)}$
}

\section{RESUMEN}

El presente trabajo realiza un análisis bibliométrico de la producción de tesis doctorales sobre hormigones defendidas en universidades españolas entre 1977 y 2019, y recogidas en la base de datos TESEO del Ministerio de Educación y Formación Profesional. El número total de tesis analizadas es 689. Se ha revisado la producción de tesis en base a los años de defensa, los idiomas de redacción, las universidades de lectura y los doctores encargados de la dirección, así como la información sobre los miembros de los tribunales evaluadores. Se concluye que el número de tesis sobre hormigones se ha incrementado considerablemente en los últimos años, destacando la Universidad Politécnica de Madrid, la Universidad Politécnica de Cataluña y la Universidad Politécnica de Valencia, como instituciones con un alto número de tesis producidas y una importante presencia de su profesorado entre los directores más productivos y los miembros de tribunales con mayor presencia.

Palabras clave: Bibliometría; Tesis doctorales; Universidades; España; Hormigón; Producción científica

\section{ABSTRACT}

The present work performs a bibliometric analysis of the production of doctoral theses on concrete defended in Spanish universities between 1977 and 2019, and collected in the TESEO database of the Ministry of Education and Professional Training. The total number of theses recovered is 689. The evolution of thesis production has been reviewed based on the years of defense, the writing languages, the reading universities, and the doctors in charge of directing, as well as the information on members of the evaluating courts. It is concluded that the number of theses on concrete has increased considerably in recent years, highlighting Universidad Politécnica de Madrid, Universidad Politécnica de Cataluña and Universidad Politécnica de Valencia as institutions with high number of theses produced and a significant presence of their lecturers among the most productive directors and members of the courts with the greatest presence.

Keywords: Bibliometrics; Doctoral theses; Universities; Spain; Concrete; Scientific production.

(*)Licenciado Geografía e Historia. Autónomo, Madrid (España).

(**)Dra. Historia del arte. Instituto de ciencias de la construcción Eduardo Torroja (IETcc-CSIC), Madrid (España).

Persona de contacto/Corresponding author: gmochonb@gmail.com (G. Mochón-Bezares)

ORCID: https://orcid.org/oooo-ooo2-4886-9237 (G. Mochón-Bezares); https://orcid.org/oooo-ooo2-1639-5022 (Á. Sorli-Rojo)

Cómo citar este artículo/Citation: Gonzalo Mochón-Bezares, Ángela Sorli-Rojo (2021). Tesis doctorales sobre hormigón en universidades españolas: un análisis bibliométrico, 1977-2019. Informes de la Construcción, 73(563): e406. https://doi.org/10.3989/ic.82495

Copyright: (c) 2021 CSIC. Este es un artículo de acceso abierto distribuido bajo los términos de la licencia de uso y distribución Creative Commons Reconocimiento 4.0 Internacional (CC BY 4.0). 


\section{INTRODUCCIÓN}

En el ámbito de las tecnologías de la construcción, el tópico o materia sobre el que más documentos se han escrito es, con gran diferencia, el hormigón (1). La investigación científica sobre el hormigón ha interesado a numerosos especialistas de diferentes campos del conocimiento, lo que ha dado como resultado numerosos trabajos en los se han analizado distintos aspectos técnicos de dicho material. El estudio de los distintos tipos de hormigones desde un punto de vista tecnológico ha generado una gran cantidad de trabajos que han sido publicados en distintos tipos documentales, en su mayoría como artículos de revistas.

Aunque se ha publicado una considerable cantidad de artículos científicos sobre hormigones, lo cierto es que dicha temática apenas ha captado la atención de los especialistas en análisis métrico de la información, pues solamente se han recuperado cinco estudios que traten de forma principal sobre dicha materia. En cuatro de los análisis bibliométricos que se han recuperado se examina de forma exclusiva uno de los siguientes tipos o aplicaciones del hormigón: hormigón segmentado como método empleado en la construcción de puentes (2); hormigón autocompactante o autoconsolidado (3); microhormigón (4); hormigón prefabricado (5); en el quinto caso se estudia el tratamiento que ha recibido el hormigón en la revista Informes de la construcción entre los años 1980 y 2018 (6). Los cinco documentos citados han basado su análisis en artículos de revistas científicas y actas de congresos científicos, obviando otros tipos documentales, lo cual supone una limitación en la visión sobre la producción científica que se puede tener sobre esa materia.

Otra forma que permite evaluar la producción científica es el análisis de las tesis doctorales. El examen de este tipo de documentos permite comprobar el grado de actividad investigadora en cualquier materia de las universidades y de su personal académico a través de la dirección de dichos trabajos académicos y de los tribunales encargados de evaluarlos. La producción de tesis doctorales en las universidades españolas ha sido analizada en numerosas ocasiones y sobre una amplia variedad temática, si bien en la mayoría de los casos se opta por materias pertenecientes a las ciencias sociales o las humanidades (7). De acuerdo a lo observado en el citado estudio y en estudios publicados con posterioridad, no se han realizado análisis métricos sobre la producción de tesis españolas acerca de tecnologías de la construcción, incluyendo entre estas aquellas que estudien los hormigones.

La práctica totalidad de los estudios métricos sobre tesis españolas han utilizado la base de datos TESEO -recurso creado y editado por el Ministerio de Educación y Formación Profesional, en la cual se recogen datos de las tesis doctorales leídas en las universidades españolas desde 1976- por ser la fuente de información más completa sobre tesis doctorales, de las cuales analizan sus temáticas principales, instituciones de lectura y personas vinculadas a las mismas, tanto en su dirección como en los tribunales que las evalúan.

El objetivo principal de este estudio es el análisis de diversos aspectos sobre la producción de tesis doctorales de universidades españolas que traten sobre los hormigones, tanto desde el punto de vista del análisis sobre su composición química como del estudio sobre el empleo de dicho material en la construcción, con el fin de comprobar cuál ha sido el grado de evolución en los parámetros analizados. El rango temporal del estudio se inicia en el año en el que se leyó la primera tesis recogida en TESEO que trata sobre hormigón (1977) y finaliza con las tesis sobre esa materia fechadas en el año 2019.

Teniendo como referencia el objetivo principal, se han establecido los siguientes objetivos específicos:

- Cuantificar la evolución en la producción de tesis a lo largo de todo el periodo estudiado, tanto en lo que se refiere a las instituciones donde han defendido sus trabajos los doctorandos como a los doctores que se han encargado de la dirección de dichos trabajos académicos. Este objetivo busca ver cuál es el rendimiento de las universidades y de los directores de tesis sobre la tecnología de los hormigones.

- Identificar los miembros de los tribunales encargados de evaluar las tesis para poder comprobar la presencia de cada uno de ellos. También se busca representar, mediante técnicas del análisis de redes sociales, la vinculación que se ha establecido entre el personal académico, bien sea como directores de las tesis o bien como miembros de los tribunales que las han evaluado.

- Observar la diferencia que se establece entre los géneros masculino y femenino en las cantidades correspondientes a los doctorandos, los directores de las tesis y los miembros de los tribunales.

\section{METODOLOGÍA}

En este trabajo se realiza un análisis bibliométrico descriptivo y longitudinal de carácter retrospectivo sobre las tesis doctorales cuya temática principal haya sido el hormigón, tanto en lo que se refiere al análisis de su composición química como a su empleo en estructuras de construcción, y que fueron defendidas en las universidades españolas durante el periodo comprendido entre los años 1977 y 2019. La principal fuente empleada en este estudio ha sido la base de datos TESEO porque es el recurso de información que mayor cantidad de datos aporta sobre las tesis doctorales que se han leído en las universidades españolas.

La base de datos TESEO proporciona más información sobre tesis doctorales que ningún otro recurso: autor, fecha, universidad, director, miembros del tribunal evaluador y descriptores de contenido tomados del Tesauro de la UNESCO (sistema de clasificación por temas que utiliza la citada base de datos). Sin embargo, en la consulta de TESEO se han observado las siguientes deficiencias, algunas de las cuales ya han sido señaladas por otros autores en anteriores trabajos sobre análisis de tesis doctorales españolas $(7,8,9)$ : datos incompletos en numerosos registros de la base de datos, falta de normalización en los nombres de personas e instituciones, limitaciones en la consulta del contenido, baja exhaustividad durante el periodo más antiguo y uso incorrecto e impreciso de las palabras clave que se han asignado a las tesis.

Para conocer las tesis sobre hormigón se realizó una consulta inicial a la base de datos TESEO empleando los descriptores que hacen referencia a dicho material: Tecnología del hormigón (código UNESCO 330505) y Hormigón pretensado (código UNESCO 330525), que se encuentran disponibles en el campo de palabras clave dentro del apartado de búsqueda avanzada. El resultado de la consulta realizada sobre estos dos descriptores fue 533 tesis. Sin embargo, durante la labor de normalización de los nombres de directores de tesis se ob- 
servaron registros de la base de datos que trataban sobre hormigones, por lo que sí resultaban pertinentes para nuestro estudio, pero en los cuales no se habían empleado ningunos de los dos descriptores mencionados más arriba. Por ello se consideró que esta forma de consulta limitaría considerablemente el número de resultados pertinentes.

Posteriormente se identificaron los departamentos universitarios que se declararon en los 533 registros recuperados con el fin de realizar una nueva búsqueda utilizándolos como elementos de interrogación, pero también se renunció a utilizar esta vía de rastreo porque se encontraron numerosos registros, especialmente entre los más antiguos, en los que no se había expresado dicha información.

Una vez desechadas ambas formas de búsqueda por lo limitados de sus resultados, se optó por realizar una búsqueda simple a través de los términos hormigón* ${ }^{*}$, cemento* o mortero* en el título y/o resumen de las tesis, restringiendo los resultados al periodo comprendido entre los años 1976 y 2019, con el objetivo de hacer una consulta de la base de datos TESEO que resultara lo más exhaustiva posible. El resultado que se obtuvo con esta última consulta fue de 1115 registros.

Los títulos y resúmenes de las referencias de esos 1115 registros fueron sometidas a revisión con el fin de incluir aquellas tesis cuya temática principal de investigación fuese el análisis químico del hormigón o el uso de este material como elemento en la construcción. De esta manera se han excluido aquellas tesis que han abordado de forma parcial esas dos temáticas o en las que se han realizado estudios sobre ese material desde otros puntos de vista como son la producción industrial o su coste económico. Tras la revisión el conjunto final de tesis que fueron sometidas a análisis fue de 689 , que es considerada una cantidad suficiente para ver las variables de dichos trabajos académicos.

Para suplir la falta de información observada en algunos registros de TESEO, y completar los resultados obtenidos con esa base de datos, se ha recurrido a otras fuentes como son la base de datos Dialnet Tesis, el repositorio cooperativo Tesis Doctorals en Xarxa (TDX) y repositorios de universidades que tienen especial relevancia en la investigación sobre el tema que trata este estudio, entre los que destacan de forma especial el Archivo Digital Universidad Politécnica de Madrid, el repositorio RiuNET de la Universitat Politècnica de Valencia y el Portal de acceso abierto al conocimiento de la Universidad Politécnica de Cataluña (UPCommons). Además, se consultaron diferentes fichas resumen de tesis doctorales recogidas en la revista Informes de la Construcción para completar datos sobre los directores de tesis.

Para realizar el análisis bibliométrico de las tesis seleccionadas se han estudiado las siguientes variables:

1. Evolución temporal de la producción documental. Se toman las fechas de lectura declaradas en los registros de la base TESEO y se agrupan por anualidades para ver cuál ha sido la trayectoria en la defensa de tesis sobre la temática estudiada durante todo el periodo considerado.

2. Idioma que se ha empleado en la redacción de cada tesis. Para comprobar la lengua de cada documento se busca su versión a texto completo. En de no disponer del texto completo de una tesis, se considera como válido el idioma en el que se presenta su título y su resumen. Al recopilar esta información se busca comprobar el grado de internacionalidad que han alcanzado las tesis sobre la materia de estudio.

3. Universidades de lectura de tesis. Se busca comprobar la productividad de cada institución.

4. Directores de tesis. Sobre estos se evalúa su productividad (números de tesis dirigidas), y el grado de colaboración que se da entre los directores (codirección).

5. Miembros de los tribunales de evaluación. Se evalúa el grado de importancia de cada uno, prestando especial atención a aquellos que ocupan la presidencia, en base a la cantidad de tribunales de los que forma parte.

Los datos sobre directores y miembros de tribunales se han expresado también utilizando técnicas de análisis de redes sociales (ARS), las cuales permiten apreciar mejor la vinculación mediante líneas o grafos que se establece entre los actores o nodos, que en este caso son los directores de tesis y los miembros de los tribunales. Se han elaborado dos redes: una en la que se representa la coparticipación en los tribunales evaluadores y otra que expresa la selección de los tribunales por parte de los directores de tesis. En la primera los grafos no están direccionados porque representan coincidencias de los sujetos en los tribunales, mientras que en la segunda los grafos si están direccionados pues representan la selección de los miembros de los tribunales por los directores de tesis. Se comentan las posiciones de los principales actores en las redes. Para una mejor visualización de las redes en ambos casos se han limitado el número de nodos en función de la cantidad de grafos que tienen asociados.

Los datos extraídos de los recursos señalados se recogieron primeramente en una hoja de cálculo y posteriormente se trasladaron a una base de datos relacional elaborada ad-hoc. Para la visualización de las redes sociales establecidas entre los directores de tesis y los miembros de los tribunales, se empleó la herramienta de software Pajek 5.o.

\section{RESULTADOS}

\subsection{Evolución temporal de la producción}

De acuerdo a la información recogida en la base de datos TESEO, el número de tesis doctorales sobre análisis de hormigones o su uso como elemento de construcción, que han sido defendidas en las universidades españolas entre los años 1977 y 2019, asciende a 689. Esto da una media anual de 16,4 tesis, cantidad con una tendencia al alza gracias a las elevadas cifras de producción que se han recogido en las anualidades más recientes.

En general se observa que en los cuarenta y tres años examinados ha habido un elevado crecimiento en la producción de tesis doctorales que tratan las materias estudiadas. Para un mejor manejo de las cifras se han agrupado estas por quinquenios, como se ve en la figura 1. Dado que no se ha podido dividir el número total de años estudiado en periodos de cinco años se decidió incluir la producción anterior a 1980, formada por dos tesis doctorales leídas en los años 1977 y 1978, dentro del primer quinquenio, con lo que queda una fase temporal algo más prolongada (1977-1984). Durante ese primer "quinquenio" se recoge el menor número de documentos de las ocho etapas temporales establecidas y con una distribución desigual, pues en el año 1981 se defendieron casi la mitad de las tesis de esa fase y en otras anualidades (1979) no se 
registró ninguna. En el segundo quinquenio (1985-1989) se duplica la producción alcanzada en el periodo 1977-1984, y se inicia una etapa de 20 años durante la cual las cifras de cada quinquenio se incrementan de forma moderada respecto a la alcanzada en la etapa anterior. Finalmente, es importante señalar que entre los quinquenios 2005-2009 y 2015-2019 se defendieron el $64,8 \%$ de las tesis consideradas, lo que les convierte en los periodos más prolíficos de nuestro estudio.

Si se considera la producción por anualidades, los años con mayores cifras de tesis son 2016 (62) y 2017 (42), y los menos productivos han resultado ser 1977 y 1978, con una sola tesis cada uno.

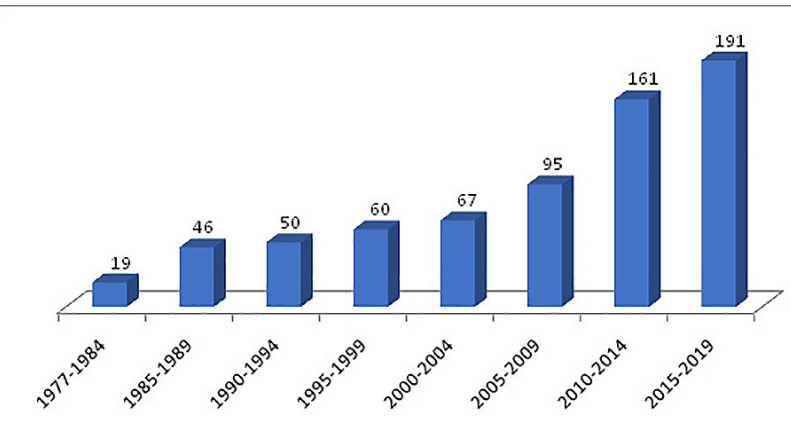

Figura 1. Producción de tesis por periodos temporales

En cuanto a la producción por género, se aprecia un porcentaje muy superior en hombres (73.6\%) que en mujeres (26,4\%). Las primeras tesis elaboradas por mujeres aparecen en 1985 , aunque al principio con una producción algo escasa: 10 tesis para un periodo de nueve años. Es desde el año 1994 cuando comienza a darse un aporte anual continuo de tesis leídas por mujeres. Desde el quinquenio 1995-1999 los porcentajes de producción para cada género han variado ligeramente (hombres $70,35 \%$ y mujeres $29,65 \%$ ), pero la diferencia continúa siendo muy grande.

\subsection{Idiomas}

La lengua más utiliza por los doctorandos para la redacción de sus tesis ha sido el castellano con un total de 606 docu- mentos (87,95\%). Se han empleado en la redacción de las tesis otras lenguas oficiales de diversos territorios españoles, aunque con una presencia meramente testimonial: se leyó una tesis en euskera en la Universidad del País Vasco en el año 2010 y otra en gallego en la Universidad de Vigo en 2011. Las restantes tesis que se han recogido están escritas en inglés (11,76\%). El uso de la lengua inglesa en la redacción de tesis se observa por primera vez en 1996 y de una forma más continuada desde 2002 en la Universidad Politécnica de Cataluña, institución en la que se han leído 35 tesis en inglés sobre nuestra materia de estudio. Otras cantidades a destacar de tesis escritas en inglés son las 13 presentadas en la Universidad Politécnica de Valencia y otras 12 en la Universidad Politécnica de Madrid.

\subsection{Producción por universidades}

En los registros recogidos de la base de datos TESEO se han identificado hasta 39 universidades en las que se han defendido tesis sobre hormigón. La incorporación de cada universidad a la lista de entidades en las que se han defendido tesis sobre la materia resultó algo lenta, pues hacia la mitad del periodo estudiado (año 1998) solamente 14 de las 39 universidades identificadas tiene al menos una tesis en la base TESEO.

Entre las instituciones de lectura destacan la Universidad Politécnica de Madrid (UPM), la Universidad Politécnica de Cataluña (UPC) y la Universidad Politécnica de Valencia (UPV), entre las cuales acumulan un 64,3\% del total, como se puede apreciar en la tabla 1 . También conviene señalar que se han identificado 27 universidades, cuyos nombres no han sido incluidos en la tabla 1, con una escasa producción (igual o menor que ocho) y que suponen un $12,77 \%$ del total.

Si se considera la producción de las instituciones tomando como base la división temporal por quinquenios, se pueden constatar algunos incrementos notables en algunas de las universidades más prolíficas. Por un lado, la Universidad Politécnica de Madrid destaca con un crecimiento temprano, posterior estabilización y nuevo aumento a partir del quinquenio 2010-2014, consiguiendo doblar el número de tesis

Tabla 1. Producción de tesis por universidades (total y por quinquenios)

\begin{tabular}{|c|c|c|c|c|c|c|c|c|c|c|}
\hline & Núm. tesis & \% tesis & $77-84$ & $85-89$ & 90-94 & 95-99 & 00-04 & 05-09 & 10-14 & $15-19$ \\
\hline Universidad Politécnica de Madrid & 192 & $27,87 \%$ & 6 & 23 & 22 & 19 & 21 & 21 & 35 & 45 \\
\hline Universidad Politécnica de Cataluña & 146 & $21,19 \%$ & 5 & 10 & 11 & 18 & 22 & 22 & 30 & 28 \\
\hline Universidad Politécnica de Valencia & 105 & $15,24 \%$ & 5 & 5 & 6 & 4 & 10 & 17 & 29 & 29 \\
\hline Universidad de Alicante & 28 & $4,06 \%$ & o & o & o & 1 & 2 & 4 & 9 & 12 \\
\hline Universidad de Cantabria & 21 & $3,05 \%$ & 2 & 2 & 3 & 5 & 1 & o & 3 & 5 \\
\hline Universidad del País Vasco & 20 & $2,90 \%$ & o & 1 & 1 & 2 & 1 & 4 & 7 & 4 \\
\hline Universidad Complutense de Madrid & 16 & $2,32 \%$ & o & 3 & 3 & 4 & 2 & 1 & 1 & 2 \\
\hline Universidad de Burgos & 16 & $2,32 \%$ & o & o & o & o & 2 & 3 & 2 & 9 \\
\hline Universidad de Granada & 16 & $2,32 \%$ & o & o & 1 & 0 & o & 2 & 6 & 7 \\
\hline Universidad Autónoma de Madrid & 15 & $2,18 \%$ & o & 1 & 1 & 2 & 1 & 4 & 3 & 3 \\
\hline Universidad de A Coruña & 14 & $2,03 \%$ & o & o & o & o & 3 & 2 & 4 & 5 \\
\hline Universidad de Castilla-La Mancha & 12 & $1,74 \%$ & o & o & o & o & o & 3 & 5 & 4 \\
\hline Otras universidades & 88 & $12,77 \%$ & 1 & 1 & 2 & 5 & 2 & 12 & 27 & 38 \\
\hline
\end{tabular}


leídas entre los años 2010 y 2019, mientras que en la Universidad Politécnica de Valencia es destacable el crecimiento en el quinquenio iniciado en 2010. Las "otras universidades" pasan de 2 a 38 tesis en los tres últimos quinquenios, destacando la Universidad de Málaga con siete tesis y la de Universidad de Vigo con seis.

\subsection{Dirección de tesis}

En el conjunto de documentos manejado ha sido posible identificar a 440 académicos e investigadores que han intervenido en la dirección de 645 tesis. En las otras 44 tesis que restan (6,39\% del total), las cuales tienen su fecha de lectura entre los años 1985 y 1992, no ha sido posible identificar a sus directores. Entre los 440 directores que se han identificado la producción se encuentra bastante diversificada: $283(64,13 \%)$ son responsables de la dirección de una única tesis, 61 (13,86\%) han dirigido dos tesis, 68 (15,45\%) han dirigido entre tres y seis tesis y 28 (6.36\%) han supervisado siete o más tesis. En cuanto al género de los directores señalar que se han contabilizado 342 hombres $(77,73 \%)$ y 98 mujeres $(22,27 \%)$.

En la tabla 2 se recogen los nombres y las instituciones de los directores que han resultado más prolíficos. La entidad que mayor presencia tiene es la Universidad Politécnica de Valencia con siete directores, entre los que destacan Pedro Serna Ros y Jorge Juan Payá Bernabeu con 19 y 16 tesis respectivamente. Otros dos organismos que se distinguen por el número de académicos son la Universidad Politécnica de Cataluña y la Universidad Politécnica de Madrid. De la primera institución encontramos seis académicos, entre los que llama la atención el catedrático Antonio Aguado de Cea por la elevada cantidad de tesis que ha dirigido o codirigido, mientras que la Universidad Complutense tiene cinco directores con una productividad algo menor que la registrada por los directores de las otras dos universidades aun siendo mayor su producción total. Esto indica que la Universidad Politécnica de Madrid, institución en la que más tesis se han defendido durante el periodo temporal estudiado, presenta las labores de dirección de tesis muy distribuida entre los académicos. Entre la tabla 2 destaca también la elevada cantidad de tesis dirigidas o codirigidas por la investigadora Carmen Andrade Perdrix, del Instituto de Ciencias de la Construcción Eduardo Torroja, quien ha realizado la mayor parte de su labor de dirección de tesis con doctorandos de tres universidades madrileñas: 7 en la Universidad Politécnica de Madrid, 5 en la Universidad Complutense y 3 en la Universidad Autónoma de Madrid.

Entre los 22 académicos e investigadores que aparecen en la tabla 2 la diferencia de género es muy acusada, pues solamente se cuentan dos mujeres: Carmen Andrade Perdrix y Amparo Moragues Terrades.

Se ha encontrado un alto grado de codirección en las tesis doctorales: $304(47,13 \%)$ trabajos tienen un sólo director, 314 $(48,68 \%)$ presentan dos y $27(4,19 \%)$ con tres. En el ámbito de este estudio la colaboración en la dirección de tesis se constata por primera vez en 1988 en la Universidad Politécnica de Cataluña por parte de los académicos Eugenio Oñate Ibáñez de Navarra y Javier Oliver Olivella, si bien no hay un uso continuado de la fórmula de la codirección para la supervisión de las tesis antes del año 1999. Durante los años posteriores la producción de tesis en codirección ha tenido ciertos altibajos hasta 2016 en que alcanza 46 tesis (40 dirigidas por dos personas y 6 dirigidas por tres).
En la codirección de tesis destacan por su cantidad las colaboraciones que han realizado las siguientes parejas de académicos de la Universidad Politécnica de Valencia: José R. Martí Vargas y Pedro Ros Serna (cinco colaboraciones); Jorge Juan Payá Bernabeu y José María Monzó Balbuena (cinco colaboraciones); José Luis Bonet Senach y Manuel Luis Romero García (cuatro colaboraciones). También hay profesorado de otras universidades que presentan un elevado número de colaboraciones, como Antonio Aguado de Cea con 27 tesis, Pedro Garcés Terradillos con 14 y Jaime Fernández Gómez con 12. En el sentido contrario se puede distinguir al catedrático José Calavera Ruiz, de la Universidad Politécnica de Madrid, el cual ha dirigido 15 tesis doctorales en solitario entre los años 1981 y 2008.

Tabla 2. Nombre y filiación institucional de directores con más de ocho tesis

\begin{tabular}{|c|c|c|}
\hline Nombre & Institución & $\begin{array}{l}\text { Núm. } \\
\text { tesis }\end{array}$ \\
\hline Aguado de Cea, Antonio & $\begin{array}{l}\text { Universidad Politécnica de } \\
\text { Cataluña }\end{array}$ & 36 \\
\hline Andrade Perdrix, Carmen & $\begin{array}{l}\text { Inst. CC. de la Construcción } \\
\text { Eduardo Torroja }\end{array}$ & 23 \\
\hline Serna Ros, Pedro & $\begin{array}{l}\text { Universidad Politécnica de } \\
\text { Valencia }\end{array}$ & 19 \\
\hline Fernández Gómez, Jaime & $\begin{array}{l}\text { Universidad Politécnica de } \\
\text { Madrid }\end{array}$ & 17 \\
\hline Garcés Terradillos, Pedro & Universidad de Alicante & 17 \\
\hline Marí Bernat, A. Ricardo & $\begin{array}{l}\text { Universidad Politécnica de } \\
\text { Cataluña }\end{array}$ & 16 \\
\hline Payá Bernabeu, J. Juan & $\begin{array}{l}\text { Universidad Politécnica de } \\
\text { Valencia }\end{array}$ & 16 \\
\hline Calavera Ruiz, José & $\begin{array}{l}\text { Universidad Politécnica de } \\
\text { Madrid }\end{array}$ & 15 \\
\hline $\begin{array}{l}\text { Fernández Cánovas, } \\
\text { Manuel }\end{array}$ & $\begin{array}{l}\text { Universidad Politécnica de } \\
\text { Madrid }\end{array}$ & 14 \\
\hline $\begin{array}{l}\text { Moragues Terrades, } \\
\text { Amparo }\end{array}$ & $\begin{array}{l}\text { Universidad Politécnica de } \\
\text { Madrid }\end{array}$ & 12 \\
\hline $\begin{array}{l}\text { Pialarissi Cavalaro, } \mathrm{S} . \\
\text { Henrique }\end{array}$ & $\begin{array}{l}\text { Universidad Politécnica de } \\
\text { Cataluña }\end{array}$ & 11 \\
\hline Bonet Senach, J. Luis & $\begin{array}{l}\text { Universidad Politécnica de } \\
\text { Valencia }\end{array}$ & 10 \\
\hline Gálvez Ruiz, J. Carlos & $\begin{array}{l}\text { Universidad Politécnica de } \\
\text { Madrid }\end{array}$ & 10 \\
\hline Vázquez Ramonich, Enric & $\begin{array}{l}\text { Universidad Politécnica de } \\
\text { Cataluña }\end{array}$ & 10 \\
\hline Monzo Balbuena, José M. & $\begin{array}{l}\text { Universidad Politécnica de } \\
\text { Valencia }\end{array}$ & 9 \\
\hline Romero García, M. Luis & $\begin{array}{l}\text { Universidad Politécnica de } \\
\text { Valencia }\end{array}$ & 9 \\
\hline $\begin{array}{l}\text { San José Lombera, J. } \\
\text { Tomás }\end{array}$ & Universidad del País Vasco & 9 \\
\hline Agulló Fité, Luis & $\begin{array}{l}\text { Universidad Politécnica de } \\
\text { Cataluña }\end{array}$ & 8 \\
\hline Casas Rius, J. Ramón & $\begin{array}{l}\text { Universidad Politécnica de } \\
\text { Cataluña }\end{array}$ & 8 \\
\hline Climent Llorca, M. Ángel & Universidad de Alicante & 8 \\
\hline Miguel Sosa, Pedro & $\begin{array}{l}\text { Universidad Politécnica de } \\
\text { Valencia }\end{array}$ & 8 \\
\hline $\begin{array}{l}\text { Valcuende Payá, M. } \\
\text { Octavio }\end{array}$ & $\begin{array}{l}\text { Universidad Politécnica de } \\
\text { Valencia }\end{array}$ & 8 \\
\hline
\end{tabular}




\subsection{Presencia en tribunales}

La información relativa a los miembros de los tribunales ha podido ser recuperada en la mayoría de los registros aportados por TESEO que se han manejado en este estudio. No obstante, en diez tesis no se ha podido averiguar ningún dato sobre los miembros de los tribunales que las han evaluado. En otros doce registros de tesis se ha constatado la falta de información sobre un miembro del tribunal.

La cifra total de doctores que han formado parte de los tribunales evaluadores es de 1186, de los cuales 763 (64,33\%) solamente han participado en un único tribunal y 155 (13,07\%) lo han hecho en dos ocasiones. El resto de los académicos identificados $(22,6 \%)$ fueron convocados a tres o más tribunales, llegando a alcanzar algunos de ellos elevadas cantidades como puede comprobarse en la tabla 3 .

Si se considera la presencia en los tribunales por el género de sus miembros, se puede apreciar que hay un porcentaje mucho mayor de hombres $(82,8 \%)$ que de mujeres $(17,2 \%)$. Aunque el periodo estudiado comienza en 1977, es a partir del año 1990 cuando se empieza a ver mujeres formando parte de los tribunales evaluadores de tesis. Si nos fijamos en la presidencia de los tribunales el porcentaje de hombres asciende a $89,81 \%$ y el de mujeres queda en $10,19 \%$. La primera mujer presidenta de un tribunal de tesis fue Amparo Moragues Terrades en el año 2000, pero no fue hasta 2008 cuando se dio una presencia continua de mujeres ocupando el cargo más importante de los tribunales evaluadores.

Según los resultados obtenidos, los académicos que han sido más demandados para formar tribunales han sido Antonio Ricardo Marí Bernat, Antonio Aguado de Cea, José Calavera Ruiz y Manuel Fernández Cánovas. El primero destaca sobre el resto porque ha formado parte del $11,78 \%$ del total de tribunales que se han sometido a análisis en este estudio. Sin embargo, su participación en el puesto de mayor prestigio, la presidencia, representa un porcentaje algo menor (40\%) que el conseguido ejerciendo como vocal en los tribunales (53,75\%). Los porcentajes más altos de presidencias ejercidas respecto del total de participación en tribunales son los obtenidos por José Antonio Torroja Cavanillas (70\%), José Calavera Ruiz (68,51\%) y Manuel Fernández Cánovas (62,26\%).

Si se consideran las instituciones de los académicos que han obtenido una mayor presencia en tribunales destaca la Universidad Politécnica de Madrid con siete doctores de los quince presentados en la tabla 3. También conviene señalar los miembros de la Universidad Politécnica de Cataluña, dos de los cuales han concentrado un gran número de participación en tribunales.

\subsection{Análisis de redes sociales}

Sobre los conjuntos reunidos de directores de tesis y miembros de tribunales se ha realizado también un análisis de la estructura social que han creado todos ellos como fruto de su actividad puesta en relación con las tesis doctorales. Los resultados de ese análisis ponen de manifiesto los vínculos que establecen a nivel personal en el ámbito académico

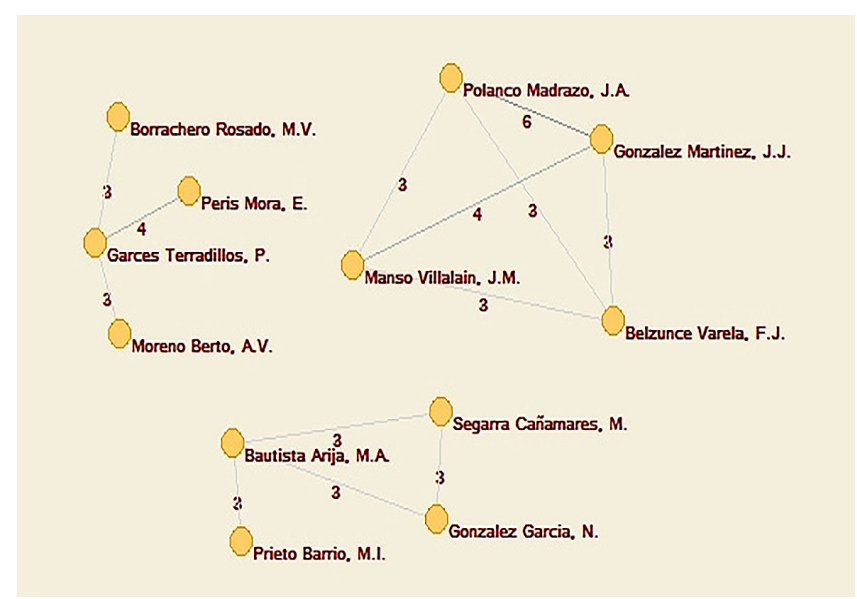

Figura 2. Red de coparticipación en tribunales (Grupos de 4 nodos)

El conjunto de miembros de los tribunales está compuesto por 1186 participantes, de los cuales 763 sólo tienen relacio-

Tabla 3. Académicos con mayor número de participaciones en tribunales

\begin{tabular}{|c|c|c|c|c|c|}
\hline Nombre & Universidad & Tribunales & Presidente & Secretario & Vocal \\
\hline Marí Bernat, Antonio Ricardo & Politécnica de Cataluña & 80 & 32 & 5 & 43 \\
\hline Aguado de Cea, Antonio & Politécnica de Cataluña & 65 & 33 & 4 & 28 \\
\hline Calavera Ruiz, José & Politécnica de Madrid & 54 & 37 & 2 & 15 \\
\hline Fernández Cánovas, Manuel & Politécnica de Madrid & 53 & 33 & 1 & 19 \\
\hline Martínez Abella, Fernando & La Coruña & 49 & 7 & 7 & 35 \\
\hline Miguel Sosa, Pedro & Politécnica de Valencia & 33 & 6 & 8 & 19 \\
\hline Corres Peiretti, Hugo & Politécnica de Madrid & 32 & 6 & 11 & 15 \\
\hline González Valle, Enrique & Politécnica de Madrid & 30 & 1 & 17 & 12 \\
\hline Torroja Cavanillas, J. Antonio & Politécnica de Madrid & 30 & 21 & O & 9 \\
\hline Villegas Cabredo, Luis & Cantabria & 30 & 4 & 4 & 22 \\
\hline Gálvez Ruiz, Jaime Carlos & Politécnica de Madrid / Castilla - La Mancha & 28 & 8 & 7 & 13 \\
\hline Vázquez Ramonich, Enric & Politécnica de Cataluña & 27 & 11 & 4 & 12 \\
\hline Serna Ros, Pedro & Politécnica de Valencia & 26 & 4 & 2 & 20 \\
\hline Fernández Prada, M. Ángel & Politécnica de Valencia & 25 & 5 & 7 & 13 \\
\hline Moragues Terrades, Amparo & Politécnica de Madrid & 20 & 5 & 4 & 11 \\
\hline
\end{tabular}




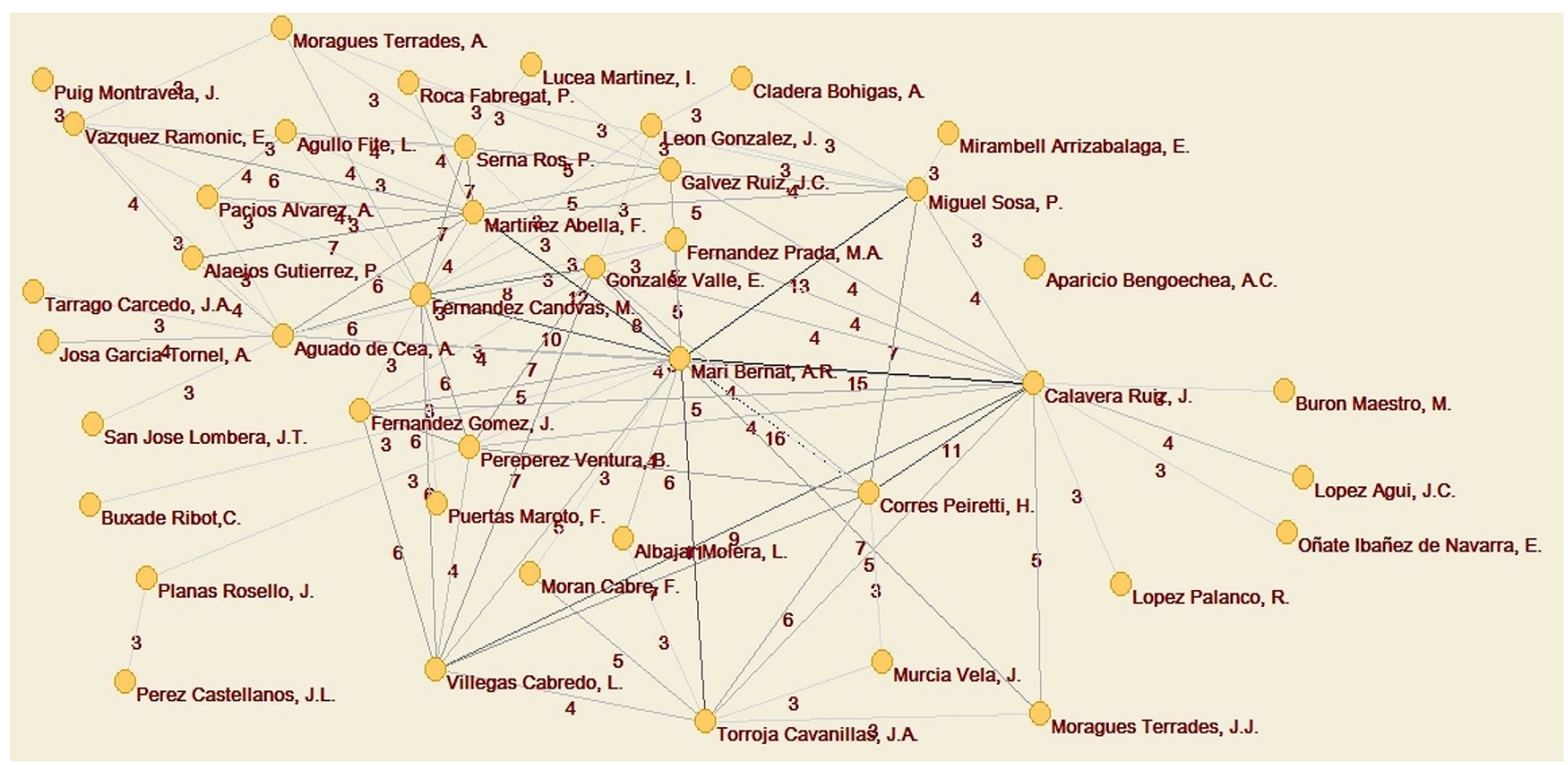

Figura 3. Red de coparticipación en tribunales (Grupo principal)

nes de valor 1. De los restantes miembros de tribunales se han tenido en cuenta los 84 miembros que presentan relaciones con otros miembros que tenga un valor tres o superior, para visualizar mejor los vínculos entre los mismos. La red obtenida presenta 4 componentes, tres de ellos más pequeños compuestos por 4 nodos que se presentan en la figura 2 y uno de mayor tamaño que agrupa a 42 actores, recogido en la figura 3 .

En los componentes de cuatro actores destacan dos por la intermediación que realizan los académicos Pedro Garcés Terradillos, que conecta elementos de universidades valencianas (Universidad Politécnica de Valencia y Universitat Jaume I) y María Asunción Bautista Arija, a través de quien se vincula a la Universidad Politécnica de Madrid con la Universidad de Castilla - La Mancha. El otro elemento de cuatro actores presenta una fuerte cohesión en su estructura pues cada uno de sus nodos está relacionado con el resto. Los actores de este componente han coincidido principalmente en tribunales de tesis defendidas en las universidades de Burgos y del País Vasco.

El componente principal de la red de miembros de tribunales está formado por 42 nodos en los que se advierten los fuertes vínculos, con un valor de diez o superior, que establece el catedrático de la Universidad Politécnica de Cataluña, Antonio Ricardo Marí Bernat, con otros cuatro académicos de la Universidad Politécnica de Madrid (José Calavera Ruiz, José Antonio Torroja, Manuel Fernández Cánovas y Hugo Corres Peiretti), uno de la Universidad Politécnica de Valencia (Pedro Miguel Sosa) y otro de la Universidad de La Coruña (Fernando Martínez Abella). Estos grafos con elevados valores conectan distintas partes de este elemento de la red y muestran tendencia a la concentración en determinados académicos.

Respecto a la relación entre miembros de tribunales y directores de tesis, se muestran en la red de las figuras 4 y 5 los académicos que han sido seleccionados al menos en cuatro ocasiones por un mismo director. Aunque en esta red los gra-

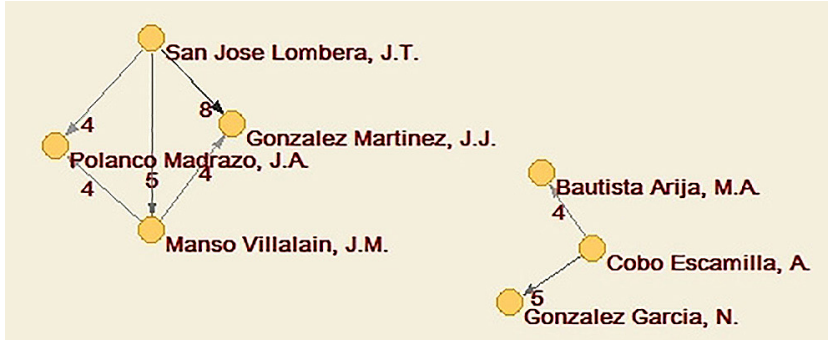

Figura 4. Red de selección de miembros de tribunales por directores (grupos de 3 y 4 nodos)

fos se encuentran direccionados, es posible que existan sujetos que seleccionen y a la vez sean seleccionados, pues actúan en algunos casos como directores y en otros como miembros de tribunales. En esta red se observan tres componentes: dos de pequeño tamaño con tres y cuatro actores respectivamente, $\mathrm{y}$ otro mayor que agrupa a 41 personas.

El componente principal de la red de miembros de tribunales está formado por 42 nodos en los que se advierten los fuertes vínculos, con un valor de diez o superior, que establece el catedrático de la Universidad Politécnica de Cataluña, Antonio Ricardo Marí Bernat, con otros cuatro académicos de la Universidad Politécnica de Madrid (José Calavera Ruiz, José Antonio Torroja, Manuel Fernández Cánovas y Hugo Corres Peiretti), uno de la Universidad Politécnica de Valencia (Pedro Miguel Sosa) y otro de la Universidad de La Coruña (Fernando Martínez Abella). Estos grafos con elevados valores conectan las distintas partes de este elemento de la red y muestran una tendencia a la concentración de los tribunales de tesis en determinados académicos.

Respecto a la relación entre miembros de tribunales y directores de tesis, se muestran en la red de las figuras 4 y 5 los académicos que han sido seleccionados al menos en cuatro ocasiones por un mismo director. Aunque en esta red los gra- 


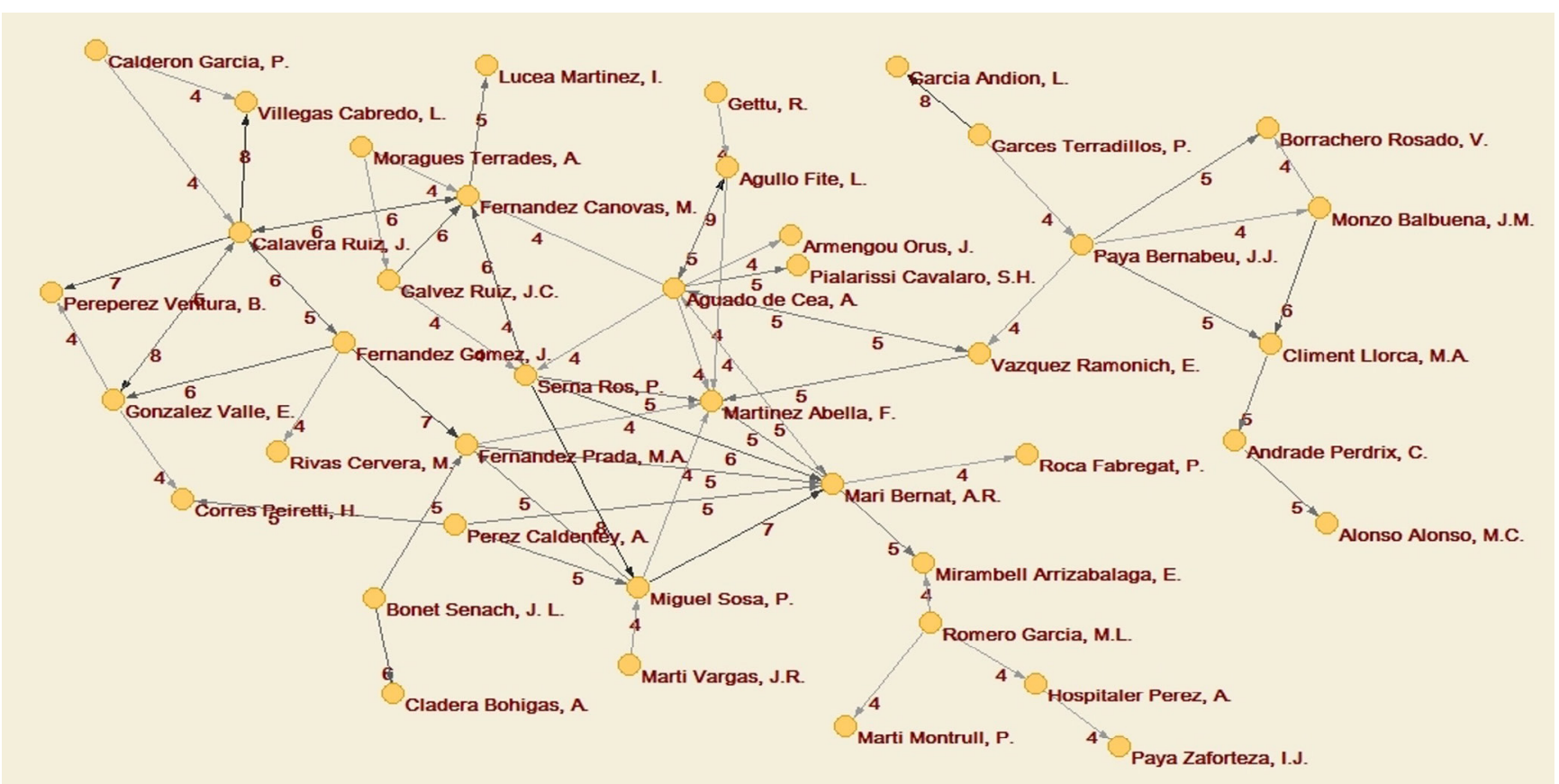

Figura 5. Red de selección de miembros de tribunales por parte de directores de tesis (grupo principal)

fos se encuentran direccionados, es posible que existan sujetos que puedan seleccionar y también ser seleccionados, pues actúan en algunos casos como directores y en otros como miembros de tribunales. En la red de selección de tribunales se observan tres componentes: dos de pequeño tamaño con tres y cuatro actores respectivamente, y otro mayor en el que se recogen 41 personas.

Los elementos pequeños, mostrados en la figura 4, evidencian la estrecha relación de un reducido grupo de doctores. El grupo de tres actores está formado por el profesor Alfonso Cobo Escamilla y otros dos académicos de universidades madrileñas a los que ha reunido hasta en cuatro ocasiones en los tribunales de tesis que ha dirigido. El elemento de cuatro actores se muestra muy cohesionado por las ocho invitaciones que ha realizado el profesor José Tomás San José Lombera a Juan Manuel Manso Villalaín, y por la preferencia que estos han mostrado porque los académicos Juan Antonio Polanco Madrazo y Javier González Martínez formasen parte de los tribunales que han evaluado a sus doctorandos.

En el elemento principal de la red relativa a la selección de tribunales destacan por su número la preferencia demostrada en nueve ocasiones por Antonio Aguado de Cea con Luis Agulló Fité, ambos de la Universidad Politécnica de Cataluña, y las ocho veces que ha convocado el catedrático José Calavera Ruiz a Enrique González del Valle, Bernardo Perepérez Ventura y Luis Villegas Cabredo. Asimismo, en la selección para formar parte de tribunales se aprecian hasta 10 casos de reciprocidad, en los que hay un alto nivel de endogamia. En tres de los casos de vínculos recíprocos está el catedrático de la Universidad Politécnica de Madrid, José Calavera Ruiz, con selecciones bidireccionales con otros tres académicos de la misma institución (Manuel Fernández Cánovas, Enrique González Valle y Jaime Fernández Gómez), y en otros tres casos se encuentra la conexión de Antonio Aguado de Cea, de la Universidad Politécnica de Cataluña, con otros tres profesores de esa misma entidad (Luis Agulló Fité, Antonio Ricardo Marí Bernat y Enric Vázquez Ramonich). Es conveniente apuntar también la cantidad de invitaciones para participar en tribunales que han recibido los académicos Fernando Martínez Abella, Manuel Fernández Cánovas y Antonio Ricardo Marí Bernat. Este último ejerce también de intermediador entre el conjunto principal de la red y varios miembros de la Universidad Politécnica de Cataluña, la Universidad Politécnica de Valencia y la Universidad Politécnica de Cartagena. Otro nodo intermediador es el correspondiente a Enric Vázquez Ramonich, que enlaza a miembros de la Universidad de Alicante, Universidad Politécnica de Valencia y el Instituto de Ciencias de la Construcción Eduardo Torroja con el resto de la red.

\section{DISCUSIÓN Y CONCLUSIONES}

La producción de tesis sobre hormigón ha ido incrementándose de manera constante desde 1977, siendo ya en la etapa final (a partir del año 2005) cuando se han contemplado los mayores aumentos entre las cifras correspondientes a cada quinquenio. Los datos recogidos sobre la realización de tesis sobre dicha materia muestran una tendencia al alza similar a la ocurrida en la producción general de tesis en España, como ya han indicado otros autores $(7,10)$. Este incremento constante nos indica que la investigación sobre hormigón que realizan los doctorandos en las universidades españolas se encuentra todavía en una fase anterior a su consolidación como objeto de estudio científico.

De acuerdo con la información recogida en las bases de datos y en los repositorios de universidades que se han consultado, el castellano es la lengua mayoritaria empleada en la redacción de las tesis. El porcentaje de trabajos en lenguas no oficiales en España, en concreto el inglés (11,74\%), puede considerarse como algo escaso. La Universidad Politécnica de Cataluña es la única entidad con un alto nivel en el aspecto lingüístico sobre la mención de doctorado internacional que se expresa en el Real Decreto 99/2011 (11), pues acumula una cantidad de tesis en inglés muy superior a las conseguidas por las otras universidades. 
En la producción por universidades se puede concluir que existe un alto grado de concentración en las tres entidades más productivas (Universidad Politécnica de Madrid, Universidad Politécnica de Cataluña y Universidad Politécnica de Valencia), aunque algunas entidades como las universidades de Alicante, Burgos y Granada, en las que se ha empezado a realizar tesis sobre hormigón en fechas más recientes, están incrementando de forma considerable su producción. Los porcentajes de producción del último quinquenio señalan que el número de tesis de las tres entidades de referencia ha descendido hasta un $53,4 \%$ del total, con lo que se puede afirmar que el grado de concentración tiende a disminuir y que se está iniciando un proceso de distribución de la producción por otras universidades.

En la dirección de tesis se constata un considerable grado de transitoriedad (64,13\%), entendido este como el número de directores que solamente han participado en la dirección de una tesis, y una cierta concentración de la producción en un reducido grupo que ha intervenido en la dirección de 293 tesis, como se puede apreciar en la tabla 2. La mayoría de los directores más productivos pertenecen a las tres universidades en la que más tesis se han leído, lo que viene a remarcar la concentración detectada al analizar las instituciones.

Por otro lado, se ha utilizado con profusión la dirección colegiada o codirección en las tesis particularmente desde la mitad del periodo estudiado, lo que ha llevado a que sea la forma de dirección más utilizada $(52,87 \%)$ frente a la fórmula de director único (47,13\%). Esto revela la existencia de una cooperación fuertemente cohesionada entre profesores universitarios en lo que a la dirección de trabajos académicos se refiere. La codirección ha sido especialmente utilizada por los académicos de la Universidad Politécnica de Valencia, la Universidad Politécnica de Madrid y la Universidad Politécnica de Cataluña.

La participación en tribunales también muestra una alta concentración en determinados miembros de las tres universidades con mayor número de tesis, muchos de los cuales son a su vez directores con una elevada producción, como puede comprobarse en las tablas 2 y 3 . El análisis de las redes sociales (figuras 2, 3, 4 y 5) sobre la coincidencia entre miembros de tribunales y la selección de tribunales por parte de los directores sirve de muestra sobre la concentración apuntada más arriba. Las cifras sobre la coincidencia en los tribunales son ciertamente elevadas y en la red sobre selección de miembros de tribunales destaca la preferencia por un pequeño número de académicos, algunos de los cuales muestran reciprocidad en las invitaciones para formar parte de tribunales.

Por último, se puede concluir también que la diferencia de género resulta muy grande en todos los aspectos estudiados. Entre los doctorandos la cantidad de mujeres comienza siendo prácticamente nula durante al principio y va incrementándose según avanza el tiempo llegando a equipararse con la producción de los hombres en los últimos años, pero la suma de todas las anualidades sólo alcanza algo más de una cuarta parte del total. Algo más escasos son los porcentajes de mujeres en dirección de tesis $(22,27 \%)$, como miembros de tribunales $(17,2 \%)$ y en la presidencia de tribunales $(10,19 \%)$. A pesar de las diferencias reseñadas hay que destacar las cifras alcanzadas en la categoría de dirección y presencia en tribunales por la catedrática Amparo Moragues Terrades y la profesora de investigación Carmen Andrade Perdrix.

\section{REFERENCIAS}

(1) Cañas-Guerrero, I., Mazarrón, F.R., Calleja-Perucho, C., Pou-Merina, A. (2014). Bibliometric analysis in the international context of the "Construction \& Building Technology" category from the Web of Science database. Construction and Building Materials, 53, 13-25. doi: https://doi.org/10.1016/j.conbuildmat.2013.10.098.

(2) Martí-Vargas, J. R., García Taengua, E., Micah Hale, W., ElBatanouny, M.E., Ziehl, P. (2015). Bibliometric analysis of Web of Science-indexed papers on concrete segmental bridges. PCI journal, 6o(1), 118-133.

(3) Mymoon, M., Mahendran, S., Lakshmi Poorna, R., Hariharan, A., Suryakala, S. (2017). Directions in Self Consolidating Concrete Research - mapping using VOSviewer. Journal of Structural Engineering (Madras), 43(5), 427-435.

(4) Silva, R.V. da, Bacarji, E., Cascudo, O. (2019). Mapeamento literário sobre o microconcreto. REEC - Revista Eletrônica De Engenharia Civil, 15(1). https://doi.org/10.5216/reec.v15i1.50421.

(5) Sánchez-Roldán, Z., Cuenca-Moyano, G.M., Martín-Morales, M., Zamorano, M. (2019). Bibliometric Study of the Use of Recycled Aggregates in the Manufacturing of Precast Concrete En J. Monteiro et al. (eds.), INCREaSE 2O19: International Congress on Engineering and Sustainability in the XXI Century (pp.964-980). Faro: Springer International Publishing. doi: https://doi.org/10.1007/978-3-030-30938-1_75.

(6) Mochón Bezares, G., Sorli Rojo, Á. (2018). Estudios sobre hormigón: su presencia a través de la revista "Informes de la construcción”. Una aproximación bibliométrica, 1980-2018. En International Conference on Construction Research Eduardo Torroja: Architecture, Engineering and Concrete/AEC 2018. 21, 22, 23 November 2018. Madrid: Fundación Eduardo Torroja; Instituto de Ciencias de la Construcción Eduardo Torroja.

(7) Fuentes Pujol, E., Argimbau Vivó, L. (2010). Las tesis doctorales en España (1997-2008): análisis, estadísticas y repositorios cooperativos. Revista Española de Documentación Científica, vol. 33(1), 63-89. doi: https://doi.org/10.3989/redc.2010.1.711.

(8) Díaz Campo, J. (2015). Análisis bibliométrico de las tesis doctorales sobre ética de los medios de comunicación presentadas en España (1979-2013). Doxa Comunicación, 20, 65-88. https://doi.org/10.31921/doxacom.n20a3.

(9) Delgado López-Cózar, E., Torres-Salinas, D., Jiménez-Contreras, E.,Ruiz-Pérez, R. (2006). Análisis bibliométrico y de redes sociales aplicado a las tesis bibliométricas defendidas en España (1976-2002): temas, escuelas científicas y redes académicas. Revista Española de Documentación Científica, 29(4), 493-524. https://doi.org/10.3989/redc.2006.v29.i4.306.

(10) Díaz-Campo, J. (2014). La investigación sobre Internet en las facultades de comunicación españolas. Análisis bibliométrico de tesis doctorales (1997-2012). Documentación de las Ciencias de la Información, 37, 305-320. doi: https://doi. org/10.5209/rev_DCIN.2014.v37.46828.

(11) Cortes generales (2011). Real Decreto 99/2011 por el que se regulan las enseñanzas oficiales de doctorado. Boletín Oficial del Estado, $\mathrm{n}^{\mathrm{O}} 35$. España. 